\title{
Generation of molecular diversity using a complexity-generating MCR-platform towards triazinane diones $\dagger$
}

\author{
Bas Groenendaal, ${ }^{a}$ Eelco Ruijter, ${ }^{a}$ Frans J. J. de Kanter, ${ }^{a}$ Martin Lutz, ${ }^{b}$ Anthony L. Spek ${ }^{b}$ and \\ Romano V. A. Orru*a
}

Received 28th April 2008, Accepted 5th June 2008

First published as an Advance Article on the web 3rd July 2008

DOI: 10.1039/b807138a

Triazinane diones, readily generated by a recently reported multicomponent reaction, can be easily alkylated with various alkyl halides, allowing a wide variety of complexity-generating secondary reactions. Because of the high variability of the initial multicomponent reactions and the multiple possibilities for participation of substituents in the secondary reactions, a highly diverse set of complex products was obtained in short and efficient reaction sequences.

\section{Introduction}

Recent advances in genomics, proteomics, metabolomics, and structural biology highlight a clear need for small molecules that can modulate biological processes. ${ }^{1}$ Combinatorial synthesis is undisputed as an enabling tool to access the required smallmolecule based compound collections. Although the benefit for drug discovery seems obvious, the actual hit rates for new drug candidates have decreased steadily over the past decade. ${ }^{2}$ It has become clear that not only the number of molecules but also the structural diversity and molecular complexity of the chosen scaffolds are key issues to address in the design of a compound library. ${ }^{3}$

Rapid generation of diverse sets of complex molecules can be achieved by employing diversity-oriented synthetic strategies in combination with complexity-generating reactions. ${ }^{4}$ Multicomponent reactions (MCRs), which combine in one pot at least three simple building blocks, ${ }^{5,6}$ provide a most powerful platform to access diversity as well as complexity in a limited number of reaction steps. Here we describe modular reaction sequences based on our previously reported MCR chemistry ${ }^{7,8}$ in combination with other common organic reactions or even with a second MCR.

${ }^{a}$ Department of Chemistry and Pharmaceutical Sciences, Vrije Universiteit Amsterdam, De Boelelaan 1083, 1081, HV, Amsterdam, The Netherlands. E-mail: orru@few.vu.nl; Fax: +3120 5987488; Tel: +31 205987447

${ }^{b}$ Bijvoet Center for Biomolecular Research, Crystal and Structural Chemistry, Utrecht University, Padualaan 8, 3584, CH, Utrecht, The Netherlands $\dagger$ Electronic supplementary information (ESI) available: ${ }^{1} \mathrm{H}$ and ${ }^{13} \mathrm{C}-\mathrm{NMR}$ spectra of all new compounds. CCDC reference number 686384. For ESI and crystallographic data in CIF or other electronic format see DOI: $10.1039 / \mathrm{b} 807138 \mathrm{a}$
The MCR platform that we chose for this work is a onepot synthetic protocol towards triazinane diones $\mathbf{1}$, a rather unexplored class of heterocyclic scaffolds (Scheme 1). ${ }^{7,8}$ The fourcomponent reaction (4CR) combines phosphonate $\mathbf{2}$, nitriles $\mathbf{3}$, aldehydes $\mathbf{4}$ and isocyanates $\mathbf{5}$ and proceeds with remarkable efficiency and flexibility. Furthermore, subsequent alkylation of 5 proved successful and allows attachment of additional synthetic handles.

Combination of this 4CR with additional complexitygenerating reactions, e.g., ring-closing metathesis (RCM), ${ }^{9}$ cycloaddition reactions (Huisgen ${ }^{10}$ or Diels-Alder ${ }^{11}$ ), or isonitrilebased MCRs (I-MCR) ${ }^{5}$ enables rapid access to highly complex (poly)heterocyclic scaffolds with pharmaceutically interesting cores.

\section{Results and discussion}

The triazinane dione 1a was chosen as the heterocyclic platform and our attention initially focused on the combination with additional MCRs. The isonitrile-based Ugi four-component (U4CR $)^{5,6,12}$ and Passerini three-component (P-3CR $)^{13}$ reactions, widely used to generate complex peptide-like products, were considered as candidate reactions to achieve fast complexity generation. Thus, as described before, ${ }^{7}$ the $4 \mathrm{CR}$ of $\mathbf{2}, \mathbf{3 a}, \mathbf{4 a}$ and 5a gave the triazinane dione 1a efficiently. The amide $\mathrm{NH}$ was alkylated $(\mathrm{NaH}$, tert-butyl 2-bromoacetate, DMF) to give tertbutyl ester 6 in $75 \%$ yield. Subsequently, removal of the tertbutyl group with TFA in $\mathrm{CH}_{2} \mathrm{Cl}_{2}$ afforded carboxylic acid 7 in quantitative yield.

The acid 7 was employed in either an U-4CR or a P-3CR (Scheme 2). The U-4CR was performed in $\mathrm{MeOH}$ at room
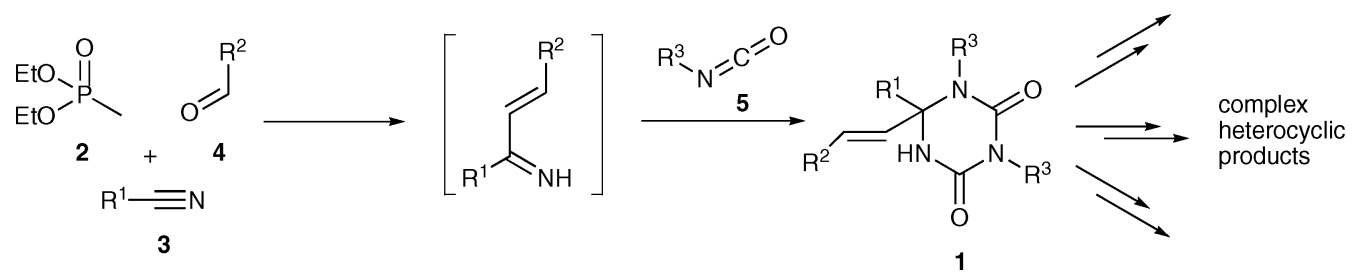

Scheme 14 CR for triazinane diones as versatile platform for complexity generation. 
<smiles>CCOP(C)(=O)OCC</smiles>

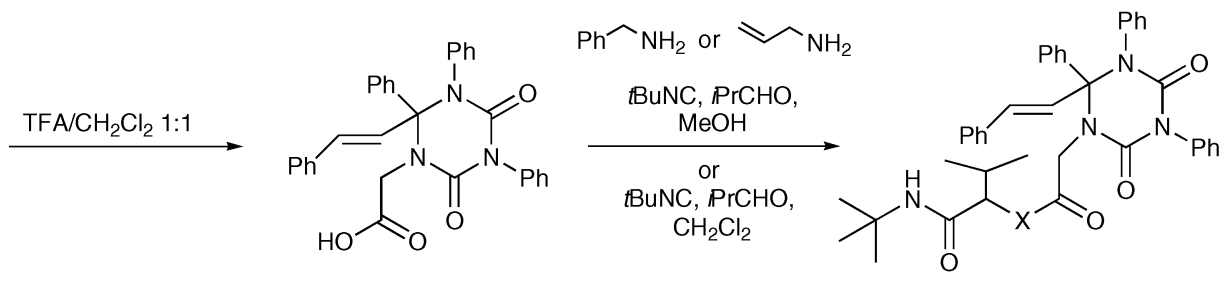

7, quant.

Scheme 2 Alkylation and then U-4CR or P-3CR reactions.

temperature using isobutyraldehyde, benzylamine or allylamine, the triazinane dione acid $\mathbf{7}$ and tert-butyl isocyanide. This indeed gave the expected peptide-derived triazinane diones $8 \mathbf{a}$ and $\mathbf{8 b}$, respectively, in reasonable to good isolated yields. The P-3CR of isobutyraldehyde, acid $\mathbf{7}$ and tert-butyl isocyanide was performed in $\mathrm{CH}_{2} \mathrm{Cl}_{2}$ at room temperature and afforded the corresponding peptide-like 9 in $62 \%$ isolated yield. Thus, combination of our $4 \mathrm{CR}$ and these isonitrile-based MCRs in a short synthetic sequence (four steps) allows for rapid construction of rather complex, peptide-functionalized heterocycles. Both the initial 4CR, which generates the heterocyclic scaffold, as well as the U-4CR and the P-3CR are easy to perform and compatible with a large variety of differently functionalized inputs. This makes this four-step sequence amenable for a combinatorial set-up to generate libraries of peptidyl triazinane diones of type $\mathbf{8}$ or $\mathbf{9}$.
Next, our attention focused on the construction of highly functionalized bi- or polycyclic ring systems. Combination of the initial triazinane dione-generating $4 \mathrm{CR}$ with $\mathrm{RCM}$ or cycloaddition reactions was envisioned as a powerful strategy to achieve this goal. Thus, allylation of $\mathbf{1 a}$ to afford $\mathbf{1 0}$ and subsequent RCM using the $2^{\text {nd }}$ generation Grubbs' catalyst ${ }^{14}$ in $\mathrm{CH}_{2} \mathrm{Cl}_{2}$ resulted in the bicyclic triazinane dione $\mathbf{1 1}(64 \%$, Scheme 3).

To further explore the potential of the triazinane dione scaffold as a versatile platform for additional cyclization reactions, a $[2+3]$ Huisgen cycloaddition (click reaction) $)^{15,16}$ was considered. Propargylation of the free $\mathrm{NH}$ in $\mathbf{1 a}(\mathrm{NaH}$, propargyl bromide, DMF; Scheme 3) gave the desired product $\mathbf{1 2}$ in $60 \%$ isolated yield. Then, the click reaction of $\mathbf{1 2}$ and readily available $\beta$ glucosyl azide derivative $\mathbf{1 3}$ was performed in a $\mathrm{H}_{2} \mathrm{O}-t-\mathrm{BuOH}-$ $\mathrm{MeCN}$ mixture with $\mathrm{CuSO}_{4}$ and sodium ascorbate as the catalyst<smiles>C#CCN1C(=O)N(c2ccccc2)C(c2ccccc2)(c2ccccc2)N(c2ccccc2)C(=O)N1c1ccccc1</smiles>

Scheme 3 Alkylation and then RCM or click reactions. 
and co-catalyst, respectively. ${ }^{15}$ The cycloaddition product $\mathbf{1 4}$ was obtained in a reasonable yield of $55 \%$. Again, combination of our initial 4CR for triazinane diones and these cyclization protocols allow rapid complexity generation in a short synthetic sequence (three steps). The RCM and the [2+3] Huisgen cycloaddition are well established and robust reactions that are compatible with a wide variety of different functionalities. This opens the way for easy generation of diversified sets of xanthine-like ${ }^{17}$ annelated bicyclic cores $\mathbf{1 1}$ or non-natural nucleoside mimics of type $14 .{ }^{18}$

Furthermore, a strategy based on the 4CR for triazinane diones and an intramolecular Diels-Alder (IMDA) reaction was envisioned to access the desired diversity and complexity of functionalized polycyclic ring systems in a highly efficient manner. ${ }^{19}$ For this purpose, we decided to introduce the required dienophile on a furan-functionalized triazinane dione (1b) platform. The four-component synthesis of $\mathbf{1 b}$ proceeded smoothly following the general procedure reported by us earlier. ${ }^{7}$ Next, reaction of $\mathbf{1 b}$ with methyl $E$-4-bromo-2-butenoate in DMF after deprotonation with $\mathrm{NaH}$ would lead to $\mathbf{1 5}$, which could then be subjected to heating to give the IMDA product. However, the anticipated IMDA reaction appears to proceed readily at room temperature and occurs immediately after alkylation of $\mathbf{1 b}$ with the dienophile. The intermediate alkylation product $\mathbf{1 5}$ was not observed. Thus, the desired polycyclic IMDA product 16a is formed in a very efficient one-pot domino process and could be isolated in $50 \%$ yield (Scheme 4). The structure of 16a including the relative stereochemistry is predicted by orbital symmetry considerations and was unambiguously confirmed by NOESY and X-ray crystal structure determination (Fig. 1).

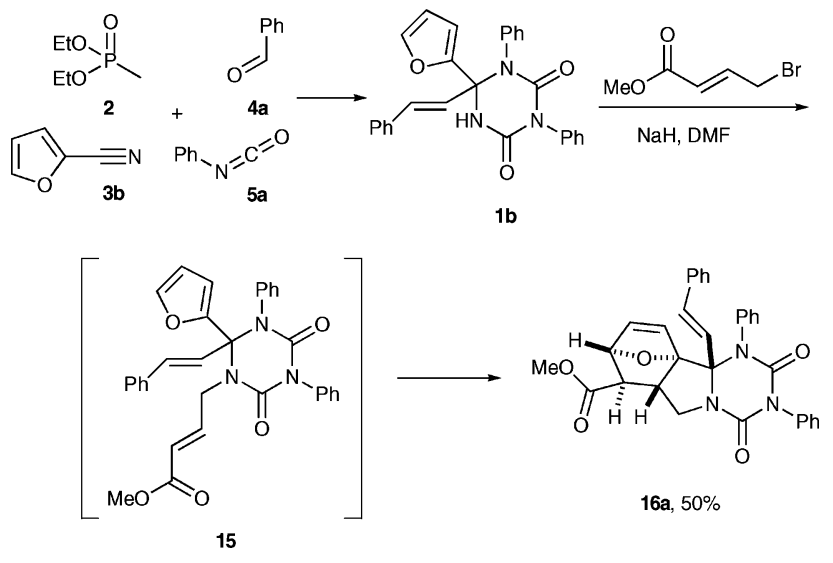

Scheme 4 A domino alkylation-IMDA reaction.

Other furan-functionalized triazinane diones underwent similar efficient spontaneous IMDA cyclization after alkylation with methyl E-4-bromo-2-butenoate. Thus, alkylation of 1c, prepared efficiently via the $4 \mathrm{CR}$ of $\mathbf{2}$, furonitrile $\mathbf{3 b}$, piperonal $\mathbf{4 b}$ and $\mathbf{5 a}$, resulted in smooth in situ IMDA cyclization to give $\mathbf{1 6} \mathbf{b}$ in high yield. Similarly, alkylation of 1d, prepared via the $4 \mathrm{CR}$ of $\mathbf{2}, \mathbf{3} \mathbf{b}$, $\mathbf{4 a}$ and $p$-methoxyphenyl isocyanate $\mathbf{5 b}$, afforded $\mathbf{1 6 c}$ (Scheme 5). The structures of $\mathbf{1 6 b}$ and $\mathbf{1 6 c}$ were assigned on the basis of NOE intensities between the hydrogens A1- $\mathbf{A 2}, \mathbf{A 1}-\mathbf{B}, \mathbf{A 2}-\mathbf{C}$ and $\mathbf{B}-\mathbf{C}$ (Fig. 1), which have a similar build-up rate as the NOE intensities

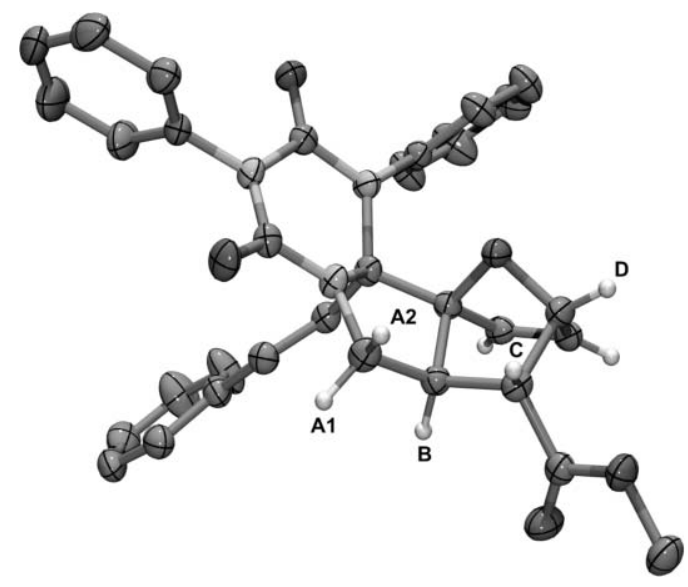

Fig. 1 Displacement ellipsoid plot of racemic 16a, drawn at the $50 \%$ probability level, indicating the stereochemistry between hydrogens $\mathbf{A 1}-\mathbf{A 2}$, A1-B, A2-C, B-C and C-D. Other hydrogen atoms are omitted for clarity.

between the corresponding hydrogens in 16a. Also, the coupling constants of the various hydrogens in $\mathbf{1 6 a}(\mathbf{A 1}-\mathbf{B}=9.5 \mathrm{~Hz} ; \mathbf{A 2}$ $\mathbf{B}=7.3 \mathrm{~Hz} ; \mathbf{B}-\mathbf{C}=2.9 \mathrm{~Hz} ; \mathbf{C}-\mathbf{D}=4.8 \mathrm{~Hz}$ ) are comparable to those observed in $\mathbf{1 6 b}$ and $\mathbf{1 6 c}$, indicating that the relative stereochemistry of all three compounds is the same.

Thus, combination of our 4CR with an alkylation-IMDA domino reaction yields a very efficient strategy to access highly functionalized polycyclic cores in only two reaction steps.

\section{Conclusions}

In summary, the $4 \mathrm{CR}$ for triazinane diones provides a versatile platform that can be applied in combination with additional MCRs, RCM, [2 + 3] cycloaddition and IMDA reactions. This results in very short reaction sequences (maximum of four) to generate both diversity and complexity. In some cases, diversification is based solely on the $N$-alkyl functionality, while in other cases various functional groups on the triazinane dione participate in the secondary reactions, thus leading to increased scaffold diversification. Combination of both approaches leads to higher overall diversity and therefore to a better coverage of chemical space. This strategy will prove useful in the design of combinatorial libraries based on highly functionalized heterocyclic small molecules.

\section{Experimental}

\section{General information}

All reactions were carried out under an inert atmosphere of dry nitrogen. THF was dried and distilled from sodium-benzophenone prior to use, $\mathrm{CH}_{2} \mathrm{Cl}_{2}$ was dried and distilled from $\mathrm{CaCl}_{2}$ prior to use. Other commercially available chemicals were used as purchased. Thin layer chromatography (TLC) was performed using aluminium TLC sheets (silica gel $60 \mathrm{~F}_{254}$ ) and compounds were visualized using UV-detection $(254 \mathrm{~nm}$ ) and colouring with an anisaldehyde solution $(6 \mathrm{~mL} p$-anisaldehyde, $7 \mathrm{~mL}$ acetic acid and $7 \mathrm{~mL}$ sulfuric acid in $120 \mathrm{~mL}$ of EtOH) or a CER-MOP solution (5 $\mathrm{g}$ molybdophosphoric acid, $2 \mathrm{~g}$ cerium(IV) sulfate and $16 \mathrm{~mL}$ sulfuric acid in $184 \mathrm{~mL}$ of $\mathrm{H}_{2} \mathrm{O}$ ). Column chromatography was performed using flash silica gel $(40-63 \mu \mathrm{m})$ and mixtures of 

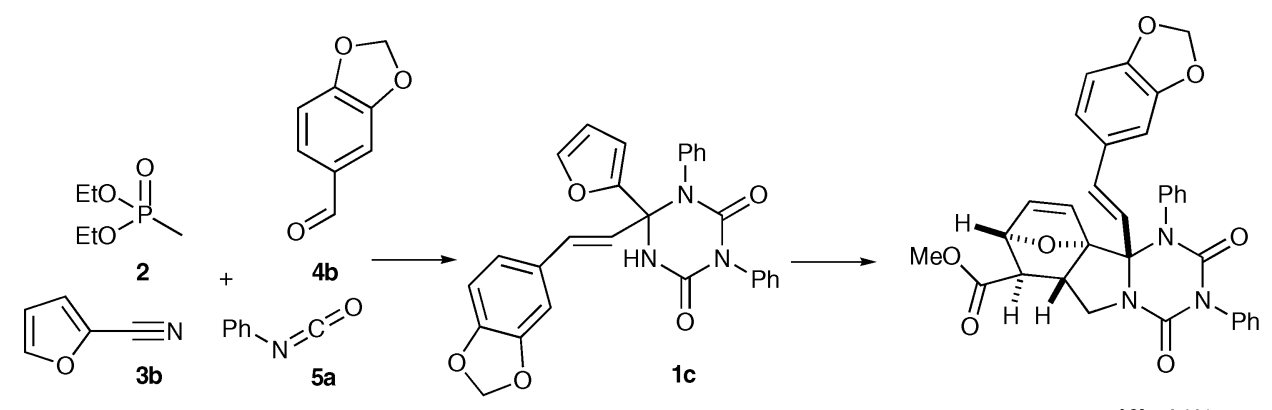

$16 b, 81 \%$

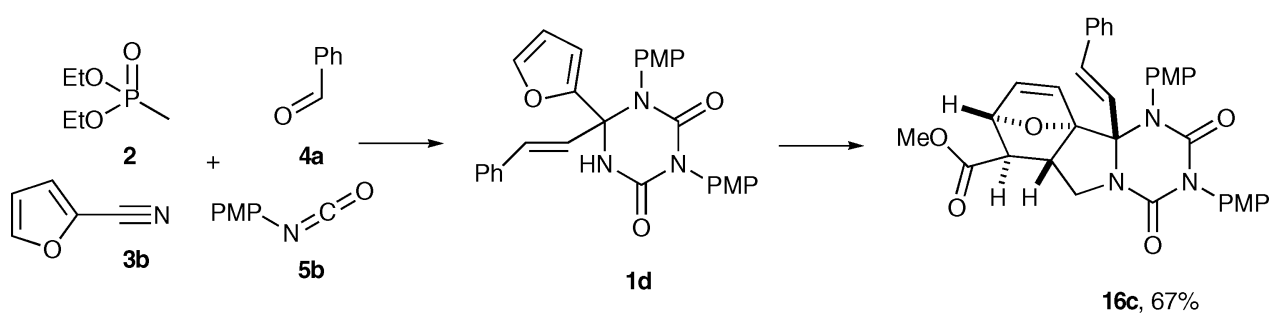

Scheme 5 Two more examples of the domino alkylation-IMDA reaction. PMP $=p$-methoxyphenyl.

cyclohexane and EtOAc. Melting points are uncorrected. Infrared (IR) spectra were obtained from pure samples and wavelengths (v) are reported in $\mathrm{cm}^{-1} \cdot{ }^{1} \mathrm{H}$ nuclear magnetic resonance (NMR) spectra were recorded at $400.13 \mathrm{MHz}$ or $250.13 \mathrm{MHz}$ and ${ }^{13} \mathrm{C}$ NMR spectra at $100.61 \mathrm{MHz}$ or $62.90 \mathrm{MHz}$ with chemical shifts $(\delta)$ reported in ppm downfield from tetramethylsilane. HRMSFAB data were measured using a four sector mass spectrometer.

\section{General procedure I: alkylation of triazinane diones}

$\mathrm{NaH}$ (1.1 equivalent, $0.12 \mathrm{M}$ ) was added to a flame-dried Schlenk vessel and dry DMF was added. This suspension was cooled to $0{ }^{\circ} \mathrm{C}$ after which the triazinane dione (1.0 equivalent. 0.11 $\mathrm{M})$ was added. The mixture was then stirred for $1.5 \mathrm{~h}$ at $0{ }^{\circ} \mathrm{C}$ after which the appropriate allylic or propargylic bromide was added (1.1 equivalent, $0.12 \mathrm{M}$ ). The reaction mixture was then allowed to warm to room temperature overnight, after which the reaction was worked up. DMF was removed by evaporation and the crude material was dissolved in EtOAc. This organic fraction was washed twice with water, then with brine, dried $\left(\mathrm{Na}_{2} \mathrm{SO}_{4}\right)$, filtrated and concentrated by evaporation of the solvent under reduced pressure. The crude product was purified by column chromatography (cyclohexane-EtOAc).

\section{tert-Butyl ester 6}

Following general procedure I, reaction between triazinane dione $1 \mathrm{a}(1.00 \mathrm{~g}, 2.20 \mathrm{mmol})$ and tert-butyl 2-bromoacetate $(340 \mathrm{mg}$, $2.40 \mathrm{mmol}$ ) followed by column chromatography (cyclohexaneEtOAc $=2: 1)$, afforded $6(945 \mathrm{mg}, 75 \%)$ as a white foam. ${ }^{1} \mathrm{H}$ NMR $\left(400 \mathrm{MHz} \mathrm{CDCl}_{3}\right): 7.45-7.58(\mathrm{~m}, 2 \mathrm{H}), 7.09-7.36(\mathrm{~m}, 18 \mathrm{H}), 6.61(\mathrm{~s}$, $2 \mathrm{H}), 4.03(\mathrm{~s}, 2 \mathrm{H}), 1.39$ (s, 9H). ${ }^{13} \mathrm{C} \mathrm{NMR}\left(101 \mathrm{MHz}, \mathrm{CDCl}_{3}\right):$ 167.7, $153.2,152.5,138.6,138.2,135.4,135.1,135.0,129.9(2 \mathrm{C}), 129.5$, 129.2 (2C), 129.0, 128.9 (2C), 128.7 (2C), 128.7 (2C), 128.6 (2C),
128.3 (2C), 128.1, 127.5, 127.0 (2C), 126.4, 81.9, 81.2, 48.2, 27.9 (3C). HRMS (FAB) calculated for $\mathrm{C}_{35} \mathrm{H}_{34} \mathrm{~N}_{3} \mathrm{O}_{4}\left(\mathrm{MH}^{+}\right)$560.2549, found 560.2543. IR (neat): 2978 (w), 1713 (s), 1676 (s), 1493 (m), 1433 (s), 1321 (m), 1229 (m), 1148 (s), 752 (s), 692 (s).

\section{Triazinane dione acid 7}

tert-Butyl ester 6 (900 mg, $1.6 \mathrm{mmol})$ was dissolved in $\mathrm{CH}_{2} \mathrm{Cl}_{2}$ $(2 \mathrm{~mL})$ and trifluoroacetic acid $(2 \mathrm{~mL})$ was added. This mixture was stirred for 45 minutes after which the solvent and excess trifluoroacetic acid were removed by evaporation under reduced pressure. This afforded crude 7 as a white solid (806 mg, quant.) without further purification being necessary. ${ }^{1} \mathrm{H} \mathrm{NMR}(250 \mathrm{MHz}$, $\left.\mathrm{CDCl}_{3}\right): 7.61-7.57(\mathrm{~m}, 2 \mathrm{H}), 7.40-7.11(\mathrm{~m}, 18 \mathrm{H}), 6.76(\mathrm{~d}, J=$ $16.0 \mathrm{~Hz}, 1 \mathrm{H}), 6.62(\mathrm{~d}, J=15.9 \mathrm{~Hz}, 1 \mathrm{H}), 4.20(\mathrm{~s}, 2 \mathrm{H}) .{ }^{13} \mathrm{C} \mathrm{NMR}$ (101 MHz, DMSO- $\left.d_{6}\right)$ : 169.5, 152.9, 152.2, 138.8, 138.5, 136.1, $135.5,134.3,130.1,129.8,129.6$ (2C), 129.3, 129.3, 129.3 (2C), 129.0 (2C), 129.0 (2C), 128.9 (2C), 128.7, 128.4, 127.6, 127.6 (2C), 127.1, 125.8, 81.5, 47.8. HRMS (FAB) calculated for $\mathrm{C}_{31} \mathrm{H}_{26} \mathrm{~N}_{3} \mathrm{O}_{4}$ $\left(\mathrm{MH}^{+}\right)$504.1923, found 504.1916. IR (neat): $2818(\mathrm{w}), 2567(\mathrm{w})$, 1784 (m), 1705 (s), 1636 (s), 1491 (m), 1460 (m), 1445 (s), 1334 (m), $1253(\mathrm{~m}), 1213$ (s), $1146(\mathrm{~s}), 756$ (s), 692 (s), 590 (s). Melting point: 181.3-181.9 ${ }^{\circ} \mathrm{C}$ (decomp.).

\section{Ugi product 8a}

Benzylamine (107 mg, $1.0 \mathrm{mmol}$ ) and isobutyraldehyde $(72 \mathrm{mg}$, $1.0 \mathrm{mmol})$ were dissolved in $\mathrm{MeOH}(5 \mathrm{~mL})$ containing $\mathrm{Na}_{2} \mathrm{SO}_{4}$ $(500 \mathrm{mg})$. This mixture was stirred for $2 \mathrm{~h}$ at room temperature, after which the acid 7 (252 $\mathrm{mg}, 0.5 \mathrm{mmol})$ was added. This mixture was then stirred for an additional 30 minutes after which tertbutyl isocyanide (42 $\mathrm{mg}, 0.5 \mathrm{mmol}$ ) was added. The reaction was stirred overnight and then worked up by addition of $\mathrm{H}_{2} \mathrm{O}$ $(25 \mathrm{~mL})$ and extraction with EtOAc $(3 \times 25 \mathrm{~mL})$. The combined organic fractions were washed with brine $(25 \mathrm{~mL})$, dried $\left(\mathrm{Na}_{2} \mathrm{SO}_{4}\right)$, 
filtered and concentrated by evaporation of the solvent under reduced pressure. The crude product was purified by column chromatography (cyclohexane-EtOAc $4: 1$, gradient) affording $\mathbf{8 a}$ (281 $\mathrm{mg}, 75 \%)$ as a white foam as a 1:1 mixture of diastereomers and rotamers. NMR spectra were recorded at $403 \mathrm{~K}$ to resolve the rotamers, but this did not have a good resolving effect on the NMR spectra. Therefore, quantification of the signals could not be achieved. ${ }^{1} \mathrm{H}$ NMR (400 MHz, DMSO- $d_{6}, 403 \mathrm{~K}$ ): 7.64 (bs), 7.57 (bs), 7.47-7.46 (m), 7.40 (bs), 7.38 (bs), 7.34-7.11 (m), 6.90$6.85(\mathrm{~m}), 6.51(\mathrm{~d}, J=16.1 \mathrm{~Hz}), 6.40(\mathrm{~d}, J=15.6 \mathrm{~Hz}), 6.26(\mathrm{~d}, J=$ $15.2 \mathrm{~Hz}), 6.23$ (d, $J=16.0 \mathrm{~Hz}), 4.85$ (bs), 4.81 (bs), 4.78 (bs), 4.74 (bs), 4.61 (bs), 4.57 (bs), 4.47 (bs), 4.44 (bs), 4.11 (bs), 2.27-2.22 (m, 1H), 1.18 (s), 1.09 (bs), 0.91 (d, $J=6.5 \mathrm{~Hz})$, 0.88 (bs), 0.74 (d, $J=6.6 \mathrm{~Hz}), 0.65$ (bs). ${ }^{13} \mathrm{C}$ NMR $(101 \mathrm{MHz}$, DMSO- $\left.d_{6}, 403 \mathrm{~K}\right): 168.5,168.4,167.8,151.8,151.8,151.4,151.4$, 139.5, 139.5, 137.9, 137.9, 135.5, 135.4, 135.3, 135.2, 134.4, 129.5, $129.5,128.3,128.3,128.0,127.9,127.8,127.8,127.8,127.7,127.1$, $127.0,126.9,126.9,126.8,126.5,126.5,126.1,125.7,80.2,80.1$, 65.3, 49.8, 49.7, 47.7, 47.6, 27.6, 27.5, 27.2, 27.1, 18.3, 18.1, 18.0. HRMS (FAB) calculated for $\mathrm{C}_{47} \mathrm{H}_{50} \mathrm{~N}_{5} \mathrm{O}_{4}\left(\mathrm{MH}^{+}\right) 748.3863$, found 748.3862. IR (neat): 3341 (w), 3065 (w), 2965 (w), 1717 (m), 1653 (s), $1491(\mathrm{~m}), 1437(\mathrm{~s}), 1302(\mathrm{~m}), 1219(\mathrm{~m}), 756(\mathrm{~m}), 711(\mathrm{~m}), 692(\mathrm{~s})$, $590(\mathrm{~m})$.

\section{Ugi product 8b}

Allylamine (145 mg, $0.75 \mathrm{mmol})$ and isobutyraldehyde $(53 \mathrm{mg}$, $0.75 \mathrm{mmol})$ were dissolved in $\mathrm{MeOH}(1 \mathrm{~mL})$ containing $\mathrm{Na}_{2} \mathrm{SO}_{4}$ $(50 \mathrm{mg})$. This mixture was stirred for $2 \mathrm{~h}$ at room temperature, after which the acid 7 (180 $\mathrm{mg}, 0.38 \mathrm{mmol})$ was added. This mixture was then stirred for an additional 30 minutes after which tertbutyl isocyanide ( $32 \mathrm{mg}, 0.38 \mathrm{mmol}$ ) was added. The reaction was stirred overnight and then worked up by addition of $\mathrm{H}_{2} \mathrm{O}$ $(10 \mathrm{~mL})$ and extraction with EtOAc $(3 \times 10 \mathrm{~mL})$. The combined organic fractions were washed with brine $(30 \mathrm{~mL})$, dried $\left(\mathrm{Na}_{2} \mathrm{SO}_{4}\right)$, filtered and concentrated by evaporation of the solvent under reduced pressure. The crude product was purified by column chromatography (cyclohexane-EtOAc $2: 1$, gradient) affording $\mathbf{8 b}$ (112 $\mathrm{mg}, 43 \%)$ as a white foam as a 1:1 mixture of diastereomers and rotamers. NMR spectra were recorded at $403 \mathrm{~K}$ to resolve the rotamers, but this did not have a good resolving effect on the NMR spectra. Therefore, quantification of the signals could not be achieved. ${ }^{1} \mathrm{H}$ NMR $\left(400 \mathrm{MHz}, \mathrm{DMSO}-d_{6}, 403 \mathrm{~K}\right): 7.68-7.65(\mathrm{~m})$, 7.52-7.39 (m), 7.33-7.28 (m), 7.24-7.16 (m), 7.08 (bs), 7.02 (bs), 6.89-6.87 (m), $6.53(\mathrm{~d}, J=16.1 \mathrm{~Hz}), 6.49(\mathrm{~d}, J=16.0 \mathrm{~Hz}), 6.32$ (d, $J=16.0 \mathrm{~Hz}$ ), $5.63(\mathrm{bs}), 5.09-5.04(\mathrm{~m}), 4.93-4.86(\mathrm{~m}), 4.66-$ $4.62(\mathrm{~m}), 4.38(\mathrm{~d}, J=17.2 \mathrm{~Hz}), 4.29(\mathrm{~d}, J=17.0 \mathrm{~Hz}), 4.04(\mathrm{~s}), 4.02$ (s), 2.19-2.14 (m), $1.23(\mathrm{~s}), 1.19$ (bs), $0.90(\mathrm{~d}, J=6.4 \mathrm{~Hz}), 0.71$ (d, $J=6.7 \mathrm{~Hz}), 0.69$ (bs). ${ }^{13} \mathrm{C}$ NMR $\left(101 \mathrm{MHz}, \mathrm{CDCl}_{3}\right): 169.6$, 169.5, 168.9, 168.8, 153.3, 153.2, 152.7, 152.7, 138.3, 138.2, 135.8, 135.4, 135.4, 135.1, 134.9, 133.6, 133.2, 130.0, 129.3, 129.1, 129.1, $129.0,128.9,128.8,128.7,128.7,128.7,128.6,128.6,128.1,128.0$, $127.5,127.4,127.2,127.1,126.9,126.9,126.8,117.1,116.9,116.8$, 81.2, 81.1, 51.3, 51.2, 47.9, 47.8, 28.5, 28.2, 26.8, 26.6, 19.5, 19.5, 18.7. HRMS (FAB) calculated for $\mathrm{C}_{43} \mathrm{H}_{48} \mathrm{~N}_{5} \mathrm{O}_{4}\left(\mathrm{MH}^{+}\right)$698.3706, found 698.3702. IR (neat): 2924 (m), $2853(\mathrm{w}), 1751$ (s), $1709(\mathrm{~m})$, $1672(\mathrm{~m}), 143(\mathrm{~m}), 1441(\mathrm{~m}), 1367(\mathrm{~m}), 1221(\mathrm{~s}), 1037(\mathrm{~s}), 912(\mathrm{~m})$, $731(\mathrm{~m}), 694(\mathrm{~m}), 596(\mathrm{w})$.

\section{Passerini product 9}

To a suspension of isobutyraldehyde $(54 \mathrm{mg}, 0.75 \mathrm{mmol})$ and 7 (252 $\mathrm{mg}, 0.50 \mathrm{mmol})$ in $\mathrm{CH}_{2} \mathrm{Cl}_{2}(5 \mathrm{~mL})$ was added tert-butyl isocyanide $(62 \mathrm{mg}, 0.75 \mathrm{mmol})$. Within a minute the reaction mixture became clear and it was then stirred overnight after which the solvent was removed by evaporation under reduced pressure. The crude product was purified by column chromatography yielding $9(203 \mathrm{mg}, 62 \%)$ as a white foam as a $1: 1$ mixture of diastereomers. Diastereomer a: ${ }^{1} \mathrm{H}$ NMR $\left(400 \mathrm{MHz}, \mathrm{CDCl}_{3}\right)$ : 7.62 $(\mathrm{dd}, J=8.1,1.9 \mathrm{~Hz}, 2 \mathrm{H}), 7.40-7.32(\mathrm{~m}, 12 \mathrm{H}), 7.15-7.10(\mathrm{~m}, 6 \mathrm{H})$, $6.86(\mathrm{~d}, J=16.0 \mathrm{~Hz}, 1 \mathrm{H}), 6.60(\mathrm{~d}, J=16.0 \mathrm{~Hz}, 1 \mathrm{H}), 6.31(\mathrm{~s}, 1 \mathrm{H})$, $4.86(\mathrm{~d}, J=3.5 \mathrm{~Hz}, 1 \mathrm{H}), 4.16(\mathrm{~d}, J=16.2 \mathrm{~Hz}, 1 \mathrm{H}), 4.00(\mathrm{~d}, J=$ $16.2 \mathrm{~Hz}, 1 \mathrm{H}), 2.34-2.27(\mathrm{~m}, 1 \mathrm{H}), 1.37$ (s, $9 \mathrm{H}), 0.89$ (d, $J=7.6 \mathrm{~Hz}$, $3 \mathrm{H}), 0.87(\mathrm{~d}, J=7.2 \mathrm{~Hz}, 3 \mathrm{H}) .{ }^{13} \mathrm{C}$ NMR $\left(101 \mathrm{MHz}, \mathrm{CDCl}_{3}\right)$ : 168.0, 167.6, 153.8, 152.0, 138.2, 137.4, 135.1, 135.1, 134.7, 129.9, 129.3, 129.2 (2C), 129.0 (2C), 129.0 (2C), 128.9 (2C), $128.8(2 \mathrm{C})$, 128.7 (2C), 128.6 (2C), 128.4, 127.5, 127.1 (2C), 125.7, 81.6, 79.4, 51.4, 48.1, 30.2, 28.3 (3C), 18.9, 16.5. Diastereomer $b:{ }^{1} \mathrm{H}$ NMR $\left(250 \mathrm{MHz}, \mathrm{CDCl}_{3}\right): 7.58-7.09(\mathrm{~m}, 20 \mathrm{H}), 6.95(\mathrm{~d}, J=16.0 \mathrm{~Hz}, 1 \mathrm{H})$, $6.81(\mathrm{~d}, J=15.9 \mathrm{~Hz}, 1 \mathrm{H}), 6.46(\mathrm{~s}, 1 \mathrm{H}), 4.93(\mathrm{~d}, J=3.2 \mathrm{~Hz}, 1 \mathrm{H}), 4.12$ $(\mathrm{d}, J=16.6 \mathrm{~Hz}, 1 \mathrm{H}), 3.94(\mathrm{~d}, J=16.6 \mathrm{~Hz}, 1 \mathrm{H}), 2.48-2.35(\mathrm{~m}, 1 \mathrm{H})$ $1.15(\mathrm{~s}, 9 \mathrm{H}), 0.99(\mathrm{~d}, J=6.9 \mathrm{~Hz}, 6 \mathrm{H}) \cdot{ }^{13} \mathrm{C} \mathrm{NMR}\left(101 \mathrm{MHz}, \mathrm{CDCl}_{3}\right)$ : 168.0, 167.7, 153.7, 152.0, 138.2, 137.3, 135.1, 134.8, 134.4, 129.9, 129.2, 129.1 (2C), 129.0 (2C), 128.9 (2C), 128.8 (2C), 128.8 (2C), 128.6 (2C), 128.5 (2C), 128.4, 127.4, 127.2 (2C), 125.8, 81.6, 79.4, 51.4, 48.5, 30.1, 28.2 (3C), 19.1, 16.4. HRMS (FAB) calculated for $\mathrm{C}_{40} \mathrm{H}_{43} \mathrm{~N}_{4} \mathrm{O}_{5}\left(\mathrm{MH}^{+}\right)$659.3233, found 659.3237. IR (neat): 3349 (w), 2967 (w), $1716(\mathrm{~s}), 1663(\mathrm{~s}), 1437(\mathrm{~s}), 1319(\mathrm{~m}), 1192(\mathrm{~m}), 756$ (s), $692(\mathrm{~s}), 588(\mathrm{~m})$.

\section{$\mathrm{N}$-Allyltriazinane dione 10}

Following general procedure I, reaction between triazinane dione 1a $(500 \mathrm{mg}, 1.12 \mathrm{mmol})$ and allyl bromide $(150 \mathrm{mg}, 1.24 \mathrm{mmol})$ followed by column chromatography (cyclohexane-EtOAc $=2$ : 1), afforded 10 (267 mg, $49 \%$ ) as a white foam. ${ }^{1} \mathrm{H}$ NMR ( $400 \mathrm{MHz}$, $\left.\mathrm{CDCl}_{3}\right): 7.57-7.55(\mathrm{~m}, 2 \mathrm{H}), 7.38-7.06(\mathrm{~m}, 18 \mathrm{H}), 6.61(\mathrm{~s}, 2 \mathrm{H}), 5.96-$ $5.89(\mathrm{~m}, 1 \mathrm{H}), 5.14$ (ddt, $J=10.2,1.4,1.4 \mathrm{~Hz}, 1 \mathrm{H}), 5.05$ (ddt, $J=$ $17.1,1.4,1.4 \mathrm{~Hz}, 1 \mathrm{H}), 4.20(\mathrm{ddt}, J=15.7,5.2,1.6 \mathrm{~Hz}, 1 \mathrm{H}), 3.98$ (ddt, $J=15.6,6.3,1.3 \mathrm{~Hz}, 1 \mathrm{H}){ }^{13} \mathrm{C}$ NMR $\left(101 \mathrm{MHz}, \mathrm{CDCl}_{3}\right)$ : $153.4,152.7,138.7,138.3,135.5,135.1,135.0,134.0,129.6(2 \mathrm{C})$, 129.4, 129.2 (2C), 129.0, 128.9 (2C), 128.7 (2C), 128.6 (4C), 128.5 (2C), 128.1, 127.3, 127.0 (2C), 126.5, 117.2, 81.7, 49.0.

HRMS (FAB) calculated for $\mathrm{C}_{32} \mathrm{H}_{28} \mathrm{~N}_{3} \mathrm{O}_{2}\left(\mathrm{MH}^{+}\right)$486.2182, found 486.2176. IR (neat): 3061 (w), 1716 (s), 1670 (s), 1491 (m), $1420(\mathrm{~s}), 1314(\mathrm{~m}), 1281(\mathrm{~m}), 754(\mathrm{~s}), 692(\mathrm{~s}), 588(\mathrm{~m})$.

\section{RCM product 11}

Grubbs' $2^{\text {nd }}$ generation catalyst $(22 \mathrm{mg}, 0.026 \mathrm{mmol})$ was added to a solution of $\mathbf{1 0}(125 \mathrm{mg}, 0.26 \mathrm{mmol})$ in $\mathrm{CH}_{2} \mathrm{Cl}_{2}(4.5 \mathrm{~mL}$, dry $)$ and this mixture was heated to reflux for $2 \mathrm{~h}$. Then the solvent was removed by evaporation under reduced pressure. The crude product was purified by column chromatography (cyclohexane$\mathrm{EtOAc}=2: 1)$ affording $11(63 \mathrm{mg}, 64 \%)$ as a grey solid. ${ }^{1} \mathrm{H}$ NMR (250 MHz, $\mathrm{CDCl}_{3}$ ): 7.49-7.28 (m, 13H), 7.15-7.12 (m, 2H), 6.25 $(\mathrm{d}, J=6.3 \mathrm{~Hz}, 1 \mathrm{H}), 5.83(\mathrm{~d}, J=6.3 \mathrm{~Hz}, 1 \mathrm{H}), 4.77(\mathrm{~d}, J=16.2 \mathrm{~Hz}$, $1 \mathrm{H}), 4.53(\mathrm{~d}, J=16.4 \mathrm{~Hz}, 1 \mathrm{H}) \cdot{ }^{13} \mathrm{C}$ NMR $\left(101 \mathrm{MHz}, \mathrm{CDCl}_{3}\right)$ : $152.5,150.6,141.1,137.5,135.3,130.2,129.3$ (2C), 129.0, 129.0 
(4C), 128.7 (2C), 128.7 (2C), 128.3, 128.2, 127.8, 125.6 (2C), 84.7, 54.0. HRMS (FAB) calculated for $\mathrm{C}_{24} \mathrm{H}_{20} \mathrm{~N}_{3} \mathrm{O}_{2}\left(\mathrm{MH}^{+}\right)$382.1556, found 382.1560. IR (neat): 3067 (w), 2872 (w), 1717 (s), 1684 (s), 1443 (s), 1319 (m), 1194 (w), 760 (m), 731 (m), 694 (m). Melting point: $199.5-200.1^{\circ} \mathrm{C}$ (decomp.).

\section{$N$-Propargyltriazinane dione 12}

Following general procedure I, reaction between triazinane dione $1 \mathrm{a}$ (500 mg, $1.12 \mathrm{mmol}$ ) and propargyl bromide (148 $\mathrm{mg}$, $1.24 \mathrm{mmol}$ ) followed by column chromatography (cyclohexaneEtOAc = 2: 1), afforded $12(326 \mathrm{mg}, 60 \%)$ as a yellow foam. ${ }^{1} \mathrm{H}$ NMR (250 MHz, $\mathrm{CDCl}_{3}$ ): 7.61-7.57 (m, 2H), 7.44-7.32 (m, $13 \mathrm{H}), 7.19-7.12(\mathrm{~m}, 5 \mathrm{H}), 6.99$ (d, $J=15.9 \mathrm{~Hz}, 1 \mathrm{H}), 6.82$ (d, $J=$ $16.0 \mathrm{~Hz}, 1 \mathrm{H}), 4.43(\mathrm{dd}, J=17.5,2.4 \mathrm{~Hz}, 1 \mathrm{H}), 3.96(\mathrm{dd}, J=$ $17.5,2.4 \mathrm{~Hz}, 1 \mathrm{H}), 2.30(\mathrm{t}, J=2.4 \mathrm{~Hz}, 1 \mathrm{H}) .{ }^{13} \mathrm{C}$ NMR $(63 \mathrm{MHz}$, $\left.\mathrm{CDCl}_{3}\right): 153.0,152.6,138.4,137.5,135.3,135.1,134.5,129.7,129.4$ (HSQC), 129.2 (2C), 129.1, 129.0 (2C), 129.0 (HSQC), 128.9 (2C), 128.8 (2C), 128.8 (2C), 128.6 (2C), 128.3, 127.3, 127.2 (2C), 126.1, 81.7, 79.8, 72.0, 35.4. HRMS (FAB) calculated for $\mathrm{C}_{32} \mathrm{H}_{26} \mathrm{~N}_{3} \mathrm{O}_{2}$ $\left(\mathrm{MH}^{+}\right)$484.2025, found 484.2032. IR (neat): $3287(\mathrm{w}), 3061(\mathrm{w})$, 1713 (s), 1674 (s), 1491 (m), 1431 (s), 1310 (m), 1281 (m), 752 (s), $691(\mathrm{~s}), 586(\mathrm{~m})$.

\section{Triazole 14}

$12(100 \mathrm{mg}, 0.21 \mathrm{mmol})$ and $\mathbf{1 3}(78 \mathrm{mg}, 0.21 \mathrm{mmol})$ were added to a mixture of $\mathrm{H}_{2} \mathrm{O}-t$-BuOH $(1: 1,400 \mu \mathrm{l}: 400 \mu \mathrm{l})$. Sodium ascorbate (17 mg, $0.084 \mathrm{mmol})$ and $\mathrm{CuSO}_{4} \cdot 5 \mathrm{H}_{2} \mathrm{O}(10 \mathrm{mg}, 0.042 \mathrm{mmol})$ were added to the white suspension and this was stirred for $2.5 \mathrm{~h}$. Then, acetonitrile $(400 \mu \mathrm{l})$ was added and the mixture was stirred overnight. An additional batch of sodium ascorbate and $\mathrm{CuSO}_{4} \cdot 5 \mathrm{H}_{2} \mathrm{O}$ were added to the clear yellow solution and the mixture was stirred for another $2 \mathrm{~h}$. The reaction was worked up by adding $\mathrm{H}_{2} \mathrm{O}(10 \mathrm{~mL})$ and extraction with $\mathrm{CH}_{2} \mathrm{Cl}_{2}(2 \times 20 \mathrm{~mL})$. The combined organic fractions were dried $\left(\mathrm{Na}_{2} \mathrm{SO}_{4}\right)$ and concentrated by evaporation of the solvent under reduced pressure. The crude product was purified by column chromatography (cyclohexaneEtOAc $=2: 1$, gradient) affording $14(99 \mathrm{mg}, 55 \%)$ as a light yellow sticky oil as a $1: 1$ mixture of diastereomers. Diastereomer $a:{ }^{1} \mathrm{H}$ NMR (400 MHz, $\left.\mathrm{CDCl}_{3}\right): 8.00(\mathrm{~s}, 1 \mathrm{H}), 7.62-7.60(\mathrm{~m}, 2 \mathrm{H})$, $7.44-7.28(\mathrm{~m}, 12 \mathrm{H}), 7.21-7.13(\mathrm{~m}, 4 \mathrm{H}), 7.08-7.06(\mathrm{~m}, 2 \mathrm{H}), 6.89(\mathrm{~d}$, $J=16.0 \mathrm{~Hz}, 1 \mathrm{H}), 6.51(\mathrm{~d}, J=15.9 \mathrm{~Hz}, 1 \mathrm{H}), 5.83-5.81(\mathrm{~m}, 1 \mathrm{H})$, $5.46-5.43(\mathrm{~m}, 2 \mathrm{H}), 5.28-5.23(\mathrm{~m}, 1 \mathrm{H}), 4.91(\mathrm{~d}, J=15.2 \mathrm{~Hz}, 1 \mathrm{H})$, 4.69 (d, $J=15.0 \mathrm{~Hz}, 1 \mathrm{H}), 4.32$ (dd, $J=12.6,4.5 \mathrm{~Hz}, 1 \mathrm{H}), 4.26-$ $4.15(\mathrm{~m}, 1 \mathrm{H}), 4.02-3.98(\mathrm{~m}, 1 \mathrm{H}), 2.10(\mathrm{~s}, 3 \mathrm{H}), 2.10$ (s, 3H), 2.08 (s, 3H), 2.04 (s, 3H). ${ }^{13} \mathrm{C} \mathrm{NMR}\left(101 \mathrm{MHz} \mathrm{CDCl}_{3}\right): 170.4,169.9$, 169.1, 168.3, 153.2, 152.4, 144.8, 139.0, 138.1, 135.7, 135.3, 135.0, 129.6 (2C), 129.3, 129.1 (2C), 128.8, 128.7 (2C), 128.6 (4C), 128.4 (2C), 128.1, 128.1 (2C), 127.3, 127.1 (2C), 127.0, 123.2, 85.7, 81.7, 75.0, 72.5, 70.3, 67.5, 61.4, 41.0, 20.6, 20.4(2C), 19.8. Diastereomer b: ${ }^{1} \mathrm{H}$ NMR (400 MHz, $\left.\mathrm{CDCl}_{3}\right): 7.89(\mathrm{~s}, 1 \mathrm{H}), 7.63-7.56(\mathrm{~m}, 2 \mathrm{H})$, 7.44-7.28 (m, 12H), 7.21-7.20 (m, 4H), 7.15-7.08 (m, 2H), 6.96 $(\mathrm{d}, J=16.0 \mathrm{~Hz}, 1 \mathrm{H}), 6.60(\mathrm{~d}, J=16.0 \mathrm{~Hz}, 1 \mathrm{H}), 5.85-5.82(\mathrm{~m}$, $1 \mathrm{H}), 5.46-5.42(\mathrm{~m}, 2 \mathrm{H}), 5.27-5.24(\mathrm{~m}, 1 \mathrm{H}), 4.82(\mathrm{~d}, J=15.2 \mathrm{~Hz}$, $1 \mathrm{H}), 4.69(\mathrm{~d}, J=15.1 \mathrm{~Hz}, 1 \mathrm{H}), 4.32(\mathrm{dd}, J=12.7,4.7 \mathrm{~Hz}, 1 \mathrm{H})$, 4.17-4.13 (m, 1H), 4.01-3.97 (m, 1H), 2.09 (s, 3H), 2.09 (s, 3H), $2.08(\mathrm{~s}, 3 \mathrm{H}), 2.05(\mathrm{~s}, 3 \mathrm{H}) .{ }^{13} \mathrm{C}$ NMR $\left(101 \mathrm{MHz}, \mathrm{CDCl}_{3}\right): 170.4$, 169.8, 169.1, 168.6, 153.4, 152.4, 144.7, 138.5, 138.1, 135.3, 135.3,
135.0, 129.6 (2C), 129.4, 129.0 (2C), 128.8, 128.7 (2C), 128.7 (2C), 128.6 (2C), 128.5 (2C), 128.3 (2C), 128.1, 127.3, 127.1 (2C), 126.9, 122.7, 85.7, 81.8, 75.0, 72.6, 70.3, 67.5, 61.3, 41.0, 20.6, 20.4 (2C), 20.0. HRMS (FAB) calculated for $\mathrm{C}_{46} \mathrm{H}_{45} \mathrm{~N}_{6} \mathrm{O}_{11}\left(\mathrm{MH}^{+}\right)$857.3146, found 857.3146. IR (neat): 3350 (w), 3067 (w), $2967(w), 2247(w)$, 1713 (s), 1655 (s), 1493 (m), 1441 (s), 1304 (m), 1223 (m), 1186 (m), 909 (s), 727 (s), 692 (s), 646 (m), 690 (m), 519 (m).

\section{Diels-Alder product 16a}

Following general procedure I, reaction between triazinane dione 1b $(250 \mathrm{mg}, 0.57 \mathrm{mmol})$ and methyl $E$-4-bromo-2-butenoate (113 $\mathrm{mg}, 0.63 \mathrm{mmol}$ ) followed by column chromatography (cyclohexane-EtOAc = $2: 1$ ), afforded 16a $(144 \mathrm{mg}, 47 \%)$ as a yellow solid. 16a was crystallized by slow evaporation of an EtOAc-solution. ${ }^{1} \mathrm{H}$ NMR (400 $\left.\mathrm{MHz}, \mathrm{CDCl}_{3}\right)$ : 7.51-7.32 (m, $12 \mathrm{H}), 7.26-7.23(\mathrm{~m}, 3 \mathrm{H}), 6.98(\mathrm{~d}, J=15.7 \mathrm{~Hz}, 1 \mathrm{H}), 6.61(\mathrm{~d}$, $J=15.8 \mathrm{~Hz}, 1 \mathrm{H}), 6.10(\mathrm{dd}, J=5.9,1.6 \mathrm{~Hz}, 1 \mathrm{H}), 6.00(\mathrm{~d}, J=$ $5.9 \mathrm{~Hz}, 1 \mathrm{H}), 5.33(\mathrm{dd}, J=4.8,1.6 \mathrm{~Hz}, 1 \mathrm{H}), 4.20(\mathrm{dd}, J=11.3$, $9.5 \mathrm{~Hz}, 1 \mathrm{H}), 3.75(\mathrm{dd}, J=11.7,7.3 \mathrm{~Hz}, 1 \mathrm{H}), 3.63(\mathrm{~s}, 3 \mathrm{H}), 3.26$ (dd, $J=4.8,2.9 \mathrm{~Hz}, 1 \mathrm{H}), 2.61$ (ddd, $J=9.4,7.3,2.9 \mathrm{~Hz}, 1 \mathrm{H})$. ${ }^{13} \mathrm{C}$ NMR (101 MHz, $\mathrm{CDCl}_{3}$ ): 170.8, 152.7, 150.5, 136.3, 135.3, 134.7, 133.8, 133.2, 133.0 (2C), 129.3 (4C), 129.1 (3C), $128.8(2 \mathrm{C})$, 128.5, 128.2, 127.2 (3C), 132.2, 98.8, 80.4, 77.1, 52.9, 52.2, 50.2, 42.7. HRMS (FAB) calculated for $\mathrm{C}_{32} \mathrm{H}_{28} \mathrm{~N}_{3} \mathrm{O}_{5}\left(\mathrm{MH}^{+}\right)$534.2029, found 534.2031.

IR (neat): 3013 (w), 1717 (s), 1676 (s), 1449 (s), 1319 (m), $1213(\mathrm{~m}), 754(\mathrm{~m}), 694(\mathrm{~m})$. Melting point: $194.9-195.4{ }^{\circ} \mathrm{C}$ (decomp.).

\section{Crystallographic data for $16 a$}

$\mathrm{C}_{32} \mathrm{H}_{27} \mathrm{~N}_{3} \mathrm{O}_{5}, \mathrm{FW}=533.57$, yellow plate, $0.36 \times 0.36 \times 0.12 \mathrm{~mm}^{3}$, monoclinic, $P 2_{1} / c$ (no. 14), $a=9.8195(1), b=25.2307$ (3), $c=$ 11.4069(2) $\AA, \beta=112.6460(5)^{\circ}, V=2608.20(6) \AA^{3}, Z=4, D_{\mathrm{x}}=$ $1.359 \mathrm{~g} \mathrm{~cm}^{-3}, \mu=0.09 \mathrm{~mm}^{-1}$. 28707 Reflections were measured on a Nonius Kappa CCD diffractometer with rotating anode (graphite monochromator, $\lambda=0.71073 \AA$ ) up to a resolution of $(\sin \theta / \lambda)_{\max }=0.65 \AA^{-1}$ at a temperature of $150 \mathrm{~K}$. The reflections were corrected for absorption and scaled on the basis of multiple measured reflections with the program SORTAV ${ }^{20}$ (0.95-0.99 correction range). 5961 Reflections were unique $\left(R_{\text {int }}=\right.$ 0.0497). The structure was solved with Direct Methods (program SHELXS-9721) and refined with SHELXL-9721 against $F^{2}$ of all reflections. Non hydrogen atoms were refined with anisotropic displacement parameters. All hydrogen atoms were located in difference Fourier maps. Methyl and phenyl hydrogen atoms were refined with a riding model; all other hydrogen atoms were refined freely with isotropic displacement parameters. 398 Parameters were refined with no restraints. $R 1 / \mathrm{w} R 2[I>2 \sigma(I)]$ : $0.0464 / 0.1247$. $R 1 /$ w $R 2$ [all refl.]: $0.0716 / 0.1416 . S=1.085$. The maximum residual electron density peak has a height of $0.84 \mathrm{e}$ $\AA^{-3}$ and a distance of $2.34 \AA$ to the closest atom H33. Geometry calculations and checking for higher symmetry was performed with the PLATON program. ${ }^{22}$

\section{Triazinane dione 1c}

Triazinane dione 1c was prepared by the method reported by Groenendaal et al. ${ }^{7}$ Reaction between diethyl methylphosphonate 
(730 mg, $5.0 \mathrm{mmol})$, furonitrile $(510 \mathrm{mg}, 5.5 \mathrm{mmol})$, piperonal (826 mg, $5.5 \mathrm{mmol})$ and phenyl isocyanate $(1.31 \mathrm{~g}, 11.0 \mathrm{mmol})$, followed by column chromatography (cyclohexane-EtOAc $4: 1$ ) afforded $1 \mathrm{c}(1.47 \mathrm{~g}, 61 \%)$ as a brown solid. ${ }^{1} \mathrm{H}$ NMR $(250 \mathrm{MHz}$, $\left.\mathrm{CDCl}_{3}\right)$ : 7.52-7.51 (m, 1H), 7.45-7.27 (m, 8H), 7.52-7.13 (m, 2H), $6.83(\mathrm{~d}, J=15.9 \mathrm{~Hz}, 1 \mathrm{H}), 6.76(\mathrm{~s}, 3 \mathrm{H}), 6.46(\mathrm{dd}, J=3.4,0.8 \mathrm{~Hz}$, $1 \mathrm{H}), 6.40(\mathrm{dd}, J=3.4,1.9 \mathrm{~Hz}, 1 \mathrm{H}), 6.11(\mathrm{~d}, J=15.8 \mathrm{~Hz}, 1 \mathrm{H}), 6.10$ (s, 1H), 5.97 (s, 2H). ${ }^{13} \mathrm{C}$ NMR (101 MHz, $\left.\mathrm{CDCl}_{3}\right)$ : 152.7, 152.0, 151.7, 148.4, 148.1, 143.5, 136.8, 134.8, 133.4, 129.9 (2C), 129.1, 129.1 (2C), 128.7 (4C), 128.2, 128.1, 123.3, 122.4, 110.7, 110.0, 108.4, 105.8, 101.3, 71.0. HRMS (FAB) calculated for $\mathrm{C}_{28} \mathrm{H}_{22} \mathrm{~N}_{3} \mathrm{O}_{5}$ $\left(\mathrm{MH}^{+}\right)$480.1559, found 480.1562. IR (neat): $3160(\mathrm{w}), 3069(\mathrm{w})$, 2899 (w), 1709 (s), 1667 (s), 1489 (m), 1444 (s), 1325 (m), 1251 (s), 1036 (s), 929 (m), 748 (m), 692 (s), 560 (s). Melting point: 205.8-206.4 ${ }^{\circ} \mathrm{C}$ (decomp.).

\section{Triazinane dione 1d}

Triazinane dione 1d was prepared by the method reported by Groenendaal et al. ${ }^{7}$ Reaction between diethyl methylphosphonate (730 mg, $5.0 \mathrm{mmol}$ ), furonitrile (510 mg, $5.5 \mathrm{mmol})$, benzaldehyde (585 $\mathrm{mg}, 5.5 \mathrm{mmol}$ ) and $p$-methoxyphenyl isocyanate $(1.64 \mathrm{~g}$, $11.0 \mathrm{mmol})$, afforded $\mathbf{1 d}(1.30 \mathrm{~g}, 52 \%)$ as a light brown solid. The crude product precipitated out of the reaction mixture after evaporation of half of the solvent and addition of water $(10 \mathrm{~mL})$. Subsequent washing of the crude product with cold $\mathrm{Et}_{2} \mathrm{O}$ gave 1d as a light brown solid. ${ }^{1} \mathrm{H}$ NMR $\left(400 \mathrm{MHz}, \mathrm{DMSO}-d_{6}\right): 9.16$ (s, $1 \mathrm{H}), 7.82(\mathrm{dd}, J=1.6,0.6 \mathrm{~Hz}, 1 \mathrm{H}), 7.46(\mathrm{~d}, J=7.2 \mathrm{~Hz}, 2 \mathrm{H})$, $7.39-7.32(\mathrm{~m}, 3 \mathrm{H}), 7.07(\mathrm{~d}, J=8.9 \mathrm{~Hz}, 2 \mathrm{H}), 7.01(\mathrm{~d}, J=8.8 \mathrm{~Hz}$, $2 \mathrm{H}), 6.95(\mathrm{~d}, J=8.9 \mathrm{~Hz}, 2 \mathrm{H}), 6.85(\mathrm{~d}, J=15.9 \mathrm{~Hz}, 1 \mathrm{H}), 6.83(\mathrm{~d}$, $J=9.0 \mathrm{~Hz}, 2 \mathrm{H}), 6.56(\mathrm{dd}, J=3.3,0.8 \mathrm{~Hz}, 1 \mathrm{H}), 6.49(\mathrm{dd}, J=$ $3.2,1.8 \mathrm{~Hz}, 1 \mathrm{H}), 6.45(\mathrm{~d}, J=16.0 \mathrm{~Hz}, 1 \mathrm{H}), 3.77(\mathrm{~s}, 3 \mathrm{H}), 3.70$ (s, $3 \mathrm{H}) .{ }^{13} \mathrm{C}$ NMR (101 MHz, DMSO- $\left.d_{6}\right): 158.9,158.7,152.7,152.4$, 152.1, 144.3, 135.6, 133.1, 131.6(2C), 130.8 (2C), 130.2, 129.1 (2C), 129.0, 128.7, 127.4 (2C), 126.6, 114.1 (4C), 111.1, 110.6, 71.0, 55.6, 55.5. HRMS (FAB) calculated for $\mathrm{C}_{29} \mathrm{H}_{26} \mathrm{~N}_{3} \mathrm{O}_{5}\left(\mathrm{MH}^{+}\right)$496.1872, found 496.1868. IR (neat): $3215(\mathrm{w}), 3065(\mathrm{w}), 2905(\mathrm{w}), 1717(\mathrm{~s})$, 1670 (s), 1508 (s), 1437 (m), 1296 (m), 1240 (s), 1028 (m), 826 (m), $743(\mathrm{~m}), 554$ (m). Melting point: $195.6-196.3{ }^{\circ} \mathrm{C}$ (decomp.).

\section{Diels-Alder product $\mathbf{1 6 b}$}

Following general procedure I, reaction between triazinane dione 1c $(250 \mathrm{mg}, 0.52 \mathrm{mmol})$ and methyl $E$-4-bromo-2-butenoate (140 mg, $0.78 \mathrm{mmol}$ ) followed by column chromatography (cyclohexane-EtOAc = $2: 1$ ), afforded 16b (150 mg, 81\%, based on recovered starting material) as an orange solid. ${ }^{1} \mathrm{H}$ NMR (400 $\mathrm{MHz}, \mathrm{CDCl}_{3}$ ): 7.47-7.29 (m, 8H), 7.23-7.20 (m, 2H), 6.98 $(\mathrm{d}, J=1.5 \mathrm{~Hz}, 1 \mathrm{H}), 6.93(\mathrm{dd}, J=8.0,1.5 \mathrm{~Hz}, 1 \mathrm{H}), 6.86(\mathrm{~d}, J=$ $16.1 \mathrm{~Hz}, 1 \mathrm{H}), 6.84(\mathrm{~d}, J=7.7 \mathrm{~Hz}, 1 \mathrm{H}), 6.41(\mathrm{~d}, J=15.6 \mathrm{~Hz}, 1 \mathrm{H})$, $6.09(\mathrm{dd}, J=5.9,1.6 \mathrm{~Hz}, 1 \mathrm{H}), 6.02(\mathrm{~s}, 2 \mathrm{H}), 5.97(\mathrm{~d}, J=5.9 \mathrm{~Hz}$, $1 \mathrm{H}), 5.32(\mathrm{dd}, J=4.8,1.5 \mathrm{~Hz}, 1 \mathrm{H}), 4.16(\mathrm{dd}, J=11.3,9.6 \mathrm{~Hz}$, $1 \mathrm{H}), 3.72(\mathrm{dd}, J=11.5,7.4 \mathrm{~Hz}, 1 \mathrm{H}), 3.62(\mathrm{~s}, 3 \mathrm{H}), 3.25$ (dd, $J=$ 4.8, $2.9 \mathrm{~Hz}, 1 \mathrm{H}), 2.58(\mathrm{ddd}, J=9.8,7.3,2.8 \mathrm{~Hz}, 1 \mathrm{H}) .{ }^{13} \mathrm{C} \mathrm{NMR}$ $\left(101 \mathrm{MHz}, \mathrm{CDCl}_{3}\right): 170.7,152.6,150.4,148.6,148.3,136.2,135.2$, 133.7, 133.1, 132.4(2C), 129.2(4C), 128.9, 128.7 (4C), 128.4, 128.0, 122.6, 121.1, 108.6, 105.8, 101.4, 98.4, 80.2, 52.8, 52.0, 50.0, 42.6. HRMS (FAB) calculated for $\mathrm{C}_{33} \mathrm{H}_{28} \mathrm{~N}_{3} \mathrm{O}_{7}\left(\mathrm{MH}^{+}\right)$578.1972, found 578.1932. IR (neat): 2955 (w), 2899 (w), 1713 (s), 1676 (s), 1449 (s),
1319 (m), 1254 (m), 1036 (m), 912 (m), 760 (m), 729 (m), 696 (m). Melting point: $161.8-162.5^{\circ} \mathrm{C}$ (decomp.)

\section{Diels-Alder product 16c}

Following general procedure I, reaction between triazinane dione 1d $(250 \mathrm{mg}, 0.50 \mathrm{mmol})$ and methyl $E$-4-bromo-2-butenoate (135 mg, $0.76 \mathrm{mmol}$ ) followed by column chromatography (cyclohexane-EtOAc = 2:1), afforded 16c (125 mg, 67\%, based on recovered starting material) as a white solid. ${ }^{1} \mathrm{H} \mathrm{NMR}(400 \mathrm{MHz}$, $\mathrm{CDCl}_{3}$ ): 7.47-7.37 (m, 6H), $7.13(\mathrm{~d}, J=8.9 \mathrm{~Hz}, 2 \mathrm{H}), 6.93$ (d, $J=$ $15.8 \mathrm{~Hz}, 1 \mathrm{H}), 6.88(\mathrm{~d}, J=9.0 \mathrm{~Hz}, 2 \mathrm{H}), 6.58(\mathrm{~d}, J=15.7 \mathrm{~Hz}$, $1 \mathrm{H}), 6.11(\mathrm{dd}, J=5.9,1.6 \mathrm{~Hz}, 1 \mathrm{H}), 5.99(\mathrm{~d}, J=5.9 \mathrm{~Hz}, 1 \mathrm{H})$, $5.33(\mathrm{dd}, J=4.9,1.6 \mathrm{~Hz}, 1 \mathrm{H}), 4.17(\mathrm{dd}, J=11.2,9.5 \mathrm{~Hz}, 1 \mathrm{H})$, $3.80(\mathrm{~s}, 3 \mathrm{H}), 3.77(\mathrm{~s}, 3 \mathrm{H}), 3.73(\mathrm{dd}, J=11.3,7.5 \mathrm{~Hz}, 1 \mathrm{H}), 3.62$ (s, 3H), $3.25(\mathrm{dd}, J=4.8,2.9 \mathrm{~Hz}, 1 \mathrm{H}), 2.56(\mathrm{ddd}, J=9.5,7.2$, $2.8 \mathrm{~Hz}, 1 \mathrm{H}) .{ }^{13} \mathrm{C}$ NMR $\left(101 \mathrm{MHz}, \mathrm{CDCl}_{3}\right): 170.7,159.2,159.0$, 153.0, 150.7, 134.7, 133.8, 133.1, 132.7 (2C), 130.1 (3C), 129.2, 128.9 (3C), 128.8, 127.9, 127.1 (3C), 123.1, 114.0 (2C), 113.5 (2C), 98.4, 80.2, 55.3, 52.8, 52.0, 50.2, 42.4. HRMS (FAB) calculated for $\mathrm{C}_{34} \mathrm{H}_{32} \mathrm{~N}_{3} \mathrm{O}_{7}\left(\mathrm{MH}^{+}\right)$594.2240, found 594.2242. IR (neat): 2955 (w), 2837 (w), 1713 (s), 1676 (s), 1510 (s), 1456 (s), 1296 (m), 1248 (s), 1169 (m), 1032 (m), 829 (m), 731 (m). Melting point: 198.7$199.3{ }^{\circ} \mathrm{C}$ (decomp.).

\section{Acknowledgements}

We thank Dr Han Peeters (University of Amsterdam) for conducting HRMS measurements. This work was financially supported by the EU and the Dutch Science Foundation (NWO/CERC3, NWO/VICI). M.L. and A.L.S. thank the Council for Chemical Sciences of the Netherlands Organization for Scientific Research (NWO-CW) for financial support.

\section{Notes and references}

1 L. Arve, T Voigt and H. Waldmann, QSAR Comb. Sci., 2006, 25, 449.

2 D. J. Newman and G. M. Cragg, J. Nat. Prod., 2007, 70, 461.

3 C. M. Dobson, Nature, 2004, 432, 824; J. Clardy and C. Walsh, Nature, 2004, 432, 829

4 M. D. Burke and S. L. Schreiber, Angew. Chem., Int. Ed., 2004, 43, 46. 5 A. Dömling, Chem. Rev., 2006, 106, 17.

6 J. Zhu, Eur. J. Org. Chem., 2003, 1133; R. V. A. Orru and M. de Greef, Synthesis, 2003, 1471.

7 B. Groenendaal, D. Vugts, R. F. Schmitz, F. J. J. de Kanter, E. Ruijter, M. B. Groen and R. V. A. Orru, J. Org. Chem., 2008, 73, 719.

8 D. J. Vugts, H. Jansen, R. F. Schmitz, F. J. J. de Kanter and R. V. A. Orru, Chem. Commun., 2003, 2594; D. J. Vugts, M. M. Koningstein, R. F. Schmitz, F. J. J. de Kanter, M. B. Groen and R. V. A. Orru, Chem.-Eur. J., 2006, 12, 7178.

9 R. H. Grubbs, Angew. Chem., Int. Ed., 2006, 45, 3760; K. C. Nicolaou, P. G. Bulger and D. Sarlah, Angew. Chem., Int. Ed., 2005, 44, 4490; A. H. Hoveyda and A. R. Zhugralin, Nature, 2007, 450, 243.

10 S. Dedola, S. A. Nepogodiev and R. A. Field, Org. Biomol. Chem., 2007, 5, 1006; M. V. Gil, M. J. Arévalo and Ó. López, Synthesis, 2007, 1589.

11 For an excellent review on furan-based Diels-Alder chemistry, see: C. O. Kappe, S. S. Murphree and A. Padwa, Tetrahedron, 1997, 53, 14179.

12 I. Ugi, R. Meyr, U. Fetzer and C. Steinbrückner, Angew. Chem., 1959, 71, 386.

13 M. Passerini, Gazz. Chim. Ital., 1921, 51, 126; L. Banfi and R. Riva, Org. React., 2005, 65, 1.

14 M. Scholl, S. Ding, C. W. Lee and R. H. Grubbs, Org. Lett., 1999, 1, 953. 
15 B. L. Wilkinson, L. F. Bornaghi, S. A. Poulsen and T. A. Houston, Tetrahedron, 2006, 62, 8115.

16 H. C. Kolb, M. G. Finn and K. B. Sharpless, Angew. Chem., Int. Ed., 2001, 40, 2004; C. W. Tornoe, C. Christensen and M. Meldal, J. Org. Chem., 2002, 67, 3057.

17 For example, see: P. G. Baraldi, F. Fruttarolo, M. A. Tabrizi, R. Romagnoli and D. Preti, Expert Opin. Drug Discovery, 2007, 2, 1161.
18 For example, see: C. Mathé and G. Gosselin, Antiviral Res., 2006, 71, 276.

19 D. Fokas, J. E. Patterson, G. Slobodkin and C. M. Baldino, Tetrahedron Lett., 2003, 44, 5137.

20 R. H. Blessing, J. Appl. Crystallogr., 1997, 30, 421.

21 G. M. Sheldrick, Acta Crystallogr., Sect. A, 2008, 64, 112.

22 A. L. Spek, J. Appl. Crystallogr., 2003, 36, 7. 